\title{
Taxonomy Classification and Comparison of Routing Protocol Based on Energy Efficient Rate
}

\author{
Dr. S. Smys, \\ Department of Computer Science and Engineering, \\ RVS Technical Campus, \\ Coimbatore, India. \\ Email: $\underline{\text { smys375@gmail.com }}$
}

\section{Dr. Abul Bashar,}

Department of Computer Engineering, Prince Mohammad Bin Fahd University, Kingdom of Saudi Arabia.

Email: abashar@pmu.edu.sa

\section{Dr. Wang Haoxiang,}

Director and lead executive faculty member,

GoPerception Laboratory,

NY, USA

Email: $\underline{\text { hw496@goperception.com }}$

\begin{abstract}
This research article investigates effective energy protocols for wireless sensor networks (WSN). The newly proposed taxonomic classification and comparison provides the following protocol categories: latency and efficient routing based on energy and hop selection in network and its architecture, communication sensor network, networking structure, procedure functioning, sending and receiving round mode, and route setting. This research work has examined each class to discuss and compare the different parameters of its representative routing protocols (mechanisms, advantages, disadvantages) based on the energy efficient rate along with delivery delay and network time. The simulation results on the NS-simulator of various protocols show that, the routing task has to be built upon different intelligent technologies to improve the network life and ensures better sensory area coverage.
\end{abstract}

Keywords: Wireless Sensor Network, Energy consumption in sensors 
Journal of ISMAC (2021)

Vol.03/ No.02

Pages: 96-110

http://irojournals.com/iroismac/

DOI: https://doi.org/10.36548/jismac.2021.2.003

\section{INTRODUCTION}

Recently, many intelligent systems are working for WSN to achieve many challenging tasks motivated by the defense and domestic applications. The WSNs are acknowledged as a new trend to capture various types of applications through the environment and physical conditions [1]. These limited features require effective system for routing task in order to increase the lifespan of communication networks [2]. Figure 1 shows the types of energy efficient routing protocol for WSN.

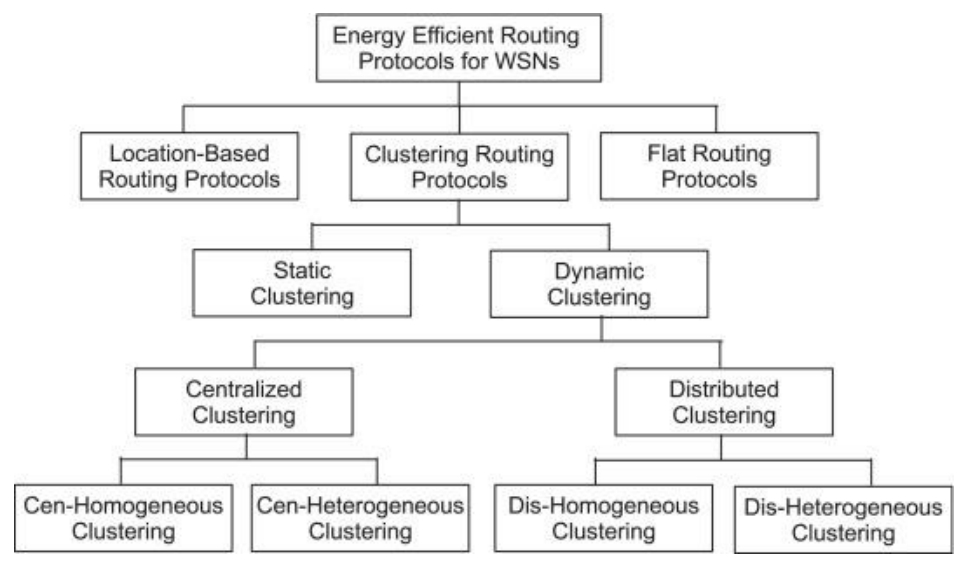

Figure 1 Types of Energy Efficient Routing Protocols of WSN

In the field of highly efficient routing protocols, much recent research attempts to solve sensor challenges, which are based on resource constraints. [3] [4]. The diverse application requirements of many sensors are required to frame a procedure for WSN requirement, which is called protocol [5-8]. Several surveys were published to analyze and classify these protocols by using various parameters. The main aim of the proposed research work is to provide an examination of the protocols under a new model of classification discussed in section 4 . Figure 2 shows the mobile sensor nodes in communication medium architecture. 


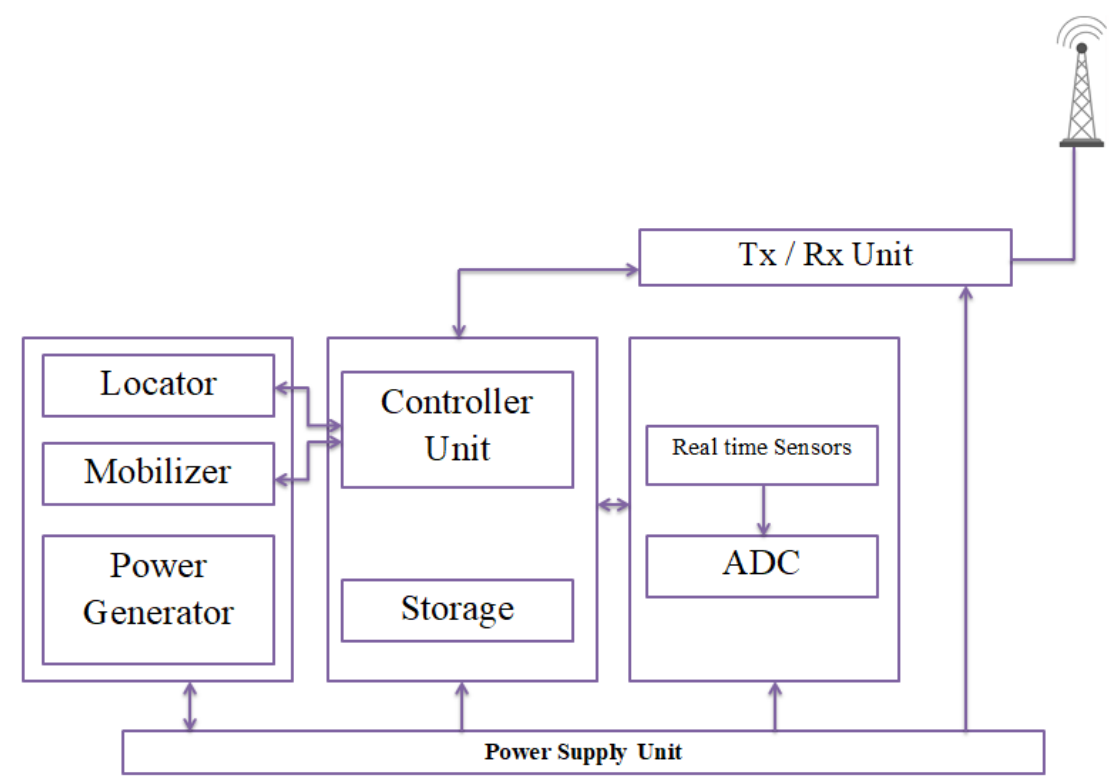

Figure 2. Mobile Sensor Node Architecture

The proposed research work has reviewed many research articles of the recently proposed protocols by using our taxonomics, identifying many protocol categories like latency and efficient routing based on energy, hop selection in network and its architecture, communication sensor network, networking structure, procedure functioning, sending and receiving round mode, and route setting [9]. We present under all categories the classification, comparison, and advantage of recent routing techniques. Displaying a short-range transmission, high sensor density, and low data rate are the principal features of WSNs [10]. Strict latency and bit error rates may also occur depending on the application. Battery substitution is a difficult task, because of the randomly distributed nature and the large density of WSNs [11]. Short battery life is an important issue that prevents the use of WSNs in different applications. Therefore, it is important to maximize the battery life of the sensor nodes. The first step is to fully understand the energy source in wireless sensor networks [12]. In this paper, an example is given to discuss the energy consumption of each hardware unit in the typical sensor node. The impact of WSN stacks on energy use is discussed by concerning the communications protocols. In addition, a survey has been conducted on existing energy consumption models. These models are classified as physical 
Journal of ISMAC (2021)

Vol.03/ No.02

Pages: 96-110

http://irojournals.com/iroismac/

DOI: https://doi.org/10.36548/jismac.2021.2.003

energy layer models, MAC energy layer models, and cross-layer energy models [13]. Network architectures like OSI and Internet, are essentially functional models organized like layers in which the layer provides the above-layer services (e.g. the application layer provides services to the end-users). The quality of its service parameters, for example, delay, flow, chipboard, availableness, reliability, and even safety are often evaluated by a network. However, concerning energy (EC), the evaluation and optimization of the network often present difficulties as a comprehensive model, which hardly takes the EC into account. Figure 3 shows some energy consumption factors of WSN [14].

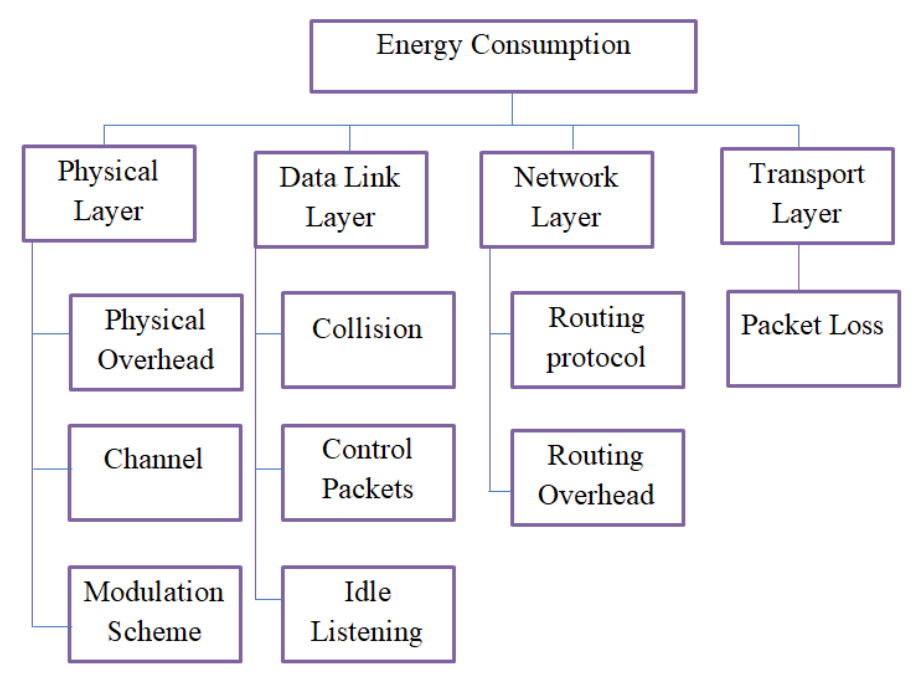

Figure 3 Energy consumption factors of WSN

In general, researchers focus on traditional network architecture, with the hope that the overall network EC is reduced without regard to other components or layers, and attempts to minimize a certain component of the single layer [15]. This isn't an ideal situation, when one doesn't know how one component fits into the entire wireless sensor network's energy picture.

\section{ORGANIZATION OF THE RESEARCH}

The remaining document is structured accordingly. Section 3 deals with the related work of WSN energy measurements. Section 4 deals with the proposed sensor node energy consumed 
Journal of ISMAC (2021)

Vol.03/ No.02

Pages: 96-110

http://irojournals.com/iroismac/

DOI: https://doi.org/10.36548/jismac.2021.2.003

in each unit with energy consumption sources individually in the communication layer. In Section 5, energy consumption in the various channel space in the channel discussed with results obtained in the simulator. Section 6 summarizes the paper and provides possible directions for future work.

\section{PRELIMINARIES}

This section is consisting of related works in detail on energy-efficient sources of WSN. The accessing of non-accessible environment can be installed by WSN sensors for many applications such as monitoring volcano, harvesting, etc. The batteries are connected with the sensors for power consumption. This battery will limit the sensors lifetime during working and makes an effective management system of WSN. The limitations are energy supply and consumption for WSN of the protocol stack [16].

Wang et al present energy minimization models for WSN to communicate the data during neglecting essential details [17]. Heinzelman et al proposed to develop the minimized power consumption model for WSN. They were computed sending and receiving data consume the energy in every single hop distance. This computation technique is saving energy at the nodes between sources to destination. They have evaluated the energy efficiency methods of WSN by power consumption model [18].

Goldsmith et al discuss traditional network drawbacks and challenges with different specifications of WSN. They created flexible network architecture for WSN with the cross-layer idea. This key idea has been proposed for enhancing the different layers. The resulting protocols are compared with protocols designed for the layer management approach. The cross-layer idea is combined for every parameter in the layers. This determination can be taken randomly to get energy-efficient [19]. Musznicki et al compute the procedures for WSN multicast with the geographic position in the communication network nodes. The implemented Dijkstra algorithm is used to find the shortest paths between sources and destination nodes in the networks. This can create the shortest energy-efficient paths through an active node in the networks. 
They concluded that these nodes of the networks are organizing within the radio range which can give good efficient energy consumptions [20]. Bhattacharya et al proposed toward source tree algorithm to produces the paths between sending data node to receive data node through the multicast tree. They focus on the number of hops that minimizing with low complexity in WSN [21].

Qiong et al presented many power consumption methods through grid and chain topologies. They compared those methods through many topologies that showed virtual connection delivery. They concluded that grid type topology meets the poorest performance in the power consumption in the networks through the main parameter [22].

\section{Proposed Work}

The proposed research work presents the routing protocol representation with two main categories based on the application:

1. Event-driven protocol

2. Time-driven protocol,

The data packets are being sent and received at regular interval after an event. Only after a weighty event is detected in the region of convergence can sense the node and send data in event-driven protocols [23] [24]. Due to various advantages, these protocols are used in several domains. The various triggered events can be immediately detected. They enable communication and the unnecessary use of energy and node computing resources to be avoided. Despite, the event-driven protocols are also available with some limitations. Since events occur in random manner, the workload is unbalanced and some nodes are activated more than the other nodes [25]. This will allow some nodes to be discharged and killed, wherein it can cause various problems, including isolated areas.

\subsection{Energy efficient routing protocol (EERP)}

The EERP protocol eliminates data redundancies by using the clustering mechanism, and it delivers best path for node to node transmission [26]. It builds expressways on a link factor to 
Journal of ISMAC (2021)

Vol.03/ No.02

Pages: 96-110

http://irojournals.com/iroismac/

DOI: https://doi.org/10.36548/jismac.2021.2.003

send the sensed data and its aggregation procedure by groups. In the study [27], researchers have shown that, this protocol provides effective power management and even accurate data aggregation detection that remains less reliable.

\subsection{Energy consumption by event protocol (ECEP)}

ECEP is developed as a reliable transmission protocol for real-time events [28]. It uses a regional mechanism for performing selection retransmission while redundancy in the data fields occurs without any network congestion. This procedure has secured the event transfer and prevents collision in the communication network since it consumes more energy.

\subsection{Prediction of Event protocol (PEP)}

PEP is designed for event monitoring applications as reliable data protocol requires quick data detection and transmission [29]. It allows nodes based on the basic decision rule and it is based on the complex network procedures to decide correctly. PEP provides the exact detection of events but it is less reliable [30].

\subsection{Data-Centric Routing Strategy (DCRS)}

The WSNs are categorized based on the routing protocol function and architectural behaviour.

1. Data-centered routing protocol

2. Position-centered route protocol

These two classes are described in the forthcoming section. By using random distributions in multitudinous sensor applications, a global identification system cannot be used to find nodes, and the traditional address-based routing is therefore not possible. The data cenetered routing protocol is based on question formation strategy which is overcome the problems in the database in queries to this environment [31]. 
Journal of ISMAC (2021)

Vol.03/ No.02

Pages: 96-110

http://irojournals.com/iroismac/

DOI: https://doi.org/10.36548/jismac.2021.2.003

\subsubsection{Active Query Mechanism (AQM)}

Basically, this mechanism involves in querying sector in the communication medium to display sensor networks for data centered routing protocol. It transfers the query and it is solving in hop at each node. This query consists of complex and passive types based on nature of the queries. AQM can handle complex queries, but in terms of energy consumption, it remains ineffective [32].

\subsubsection{Security routing in sensor network (SRSN)}

SRSN provides the secured routing in the network and it is based on a data-centered protocol for routing [33]. The routing information is collected based on the previous event query through the communication node in network, which contains real-time data and probabilistic procedure based on the fingerprint security.

\subsection{Position Centric Routing Strategy (PCRS)}

The position can be accessed by GPS technology and it is based on the signal strength of the reception signal. The transmission metrics is obtained for transmitting requests to that region and transferring data to the located area in order to reduce transmission [34].

\subsubsection{Least Hop Usage in Routing Protocol (LHURP)}

LHURP is designed as an energy-constrained routing protocol based on the location. It sends packages based on two phases they are, minimum energy consumption transmission and routing. During the transmission process, least number of hops constructs routing tables to build the finest route towards the endpoint, the shorter route to transmit envelopes to the endpoint during the routing phase. This protocol increases energy consumption by taking a short distance, where the nodes exist between active and isolated areas [35].

\subsubsection{Energy Computation in Route Protocol (ECRP)}

ECRP has been conceived as a protocol based on location. The mapping of the network energy standards globally use a global viewing mechanism [36]. The selection of transmission 
routes is based on the distribution of energy along the route and the selection with a balance in energy and this will be increased to prevent energy holes and it is improving the network lifecycle.

\section{RESULTS \& DISCUSSION}

The performance of WSN can be measured with a constant sensing area along with the constant number of sensors for evaluation and this is designed under the NS simulator [37]. In this research article, several metrics are used to measure the system efficiency and energy consumption in the networks [38]. The proposed survey comprises of transmission energy passing through several medium and paths as follows;

1. Free space channel

2. Multipath channel

$$
\begin{aligned}
& E_{T x}(k, d)=k *\left(E_{\text {elec }}+\sigma_{f S} * d^{2}\right), d \leq T_{d} \\
& E_{T x}(k, d)=k *\left(E_{\text {elec }}+\sigma_{m s} * d^{4}\right), d>T_{d}
\end{aligned}
$$

The Td is denoted as threshold distance value.

$$
T_{d}=\sqrt[2]{\sigma_{f s} / \sigma_{m s}}
$$

Where,

$\mathrm{k}$ is to be transmissible bits between source to destination

$E_{\text {elec }}$ is transmission energy in the network

$\sigma_{f s}$ is energy consumption at free space (fs) channel

$\sigma_{m s}$ is energy consumption at multi path (mp) channel

$\mathrm{d}$ denotes the distance between the nodes

The measuring metrics are tabulated in the table 1. Particularly, we concentrated on energy efficiency, Delivery delay and network life time with reliability. The results are tabulated here and it's obtained from the simulator. Figure 4 shows variable graphs between energy efficiency vs. proposed protocol. 
Journal of ISMAC (2021)

Vol.03/ No.02

Pages: 96-110

http://irojournals.com/iroismac/

DOI: https://doi.org/10.36548/jismac.2021.2.003

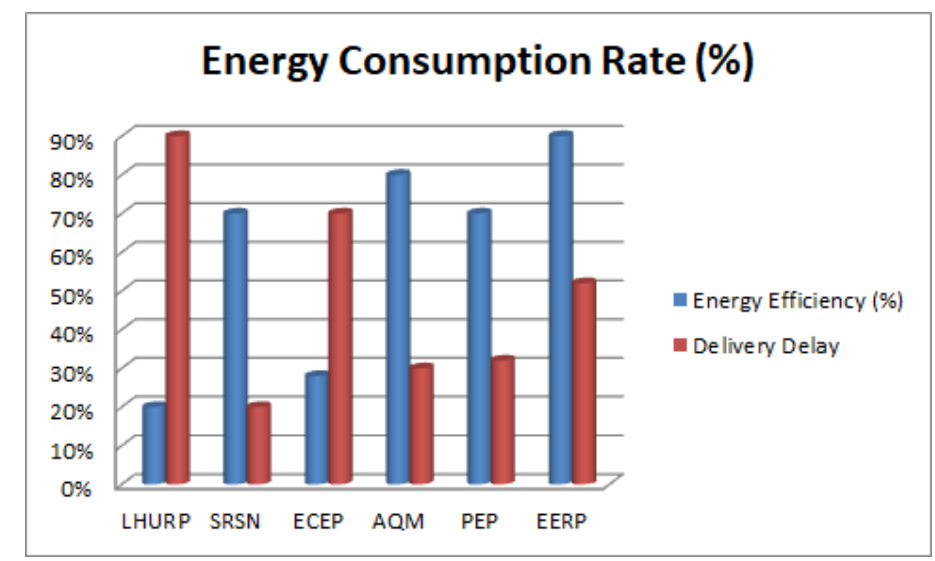

Figure 4 Energy Efficiency Vs Protocol

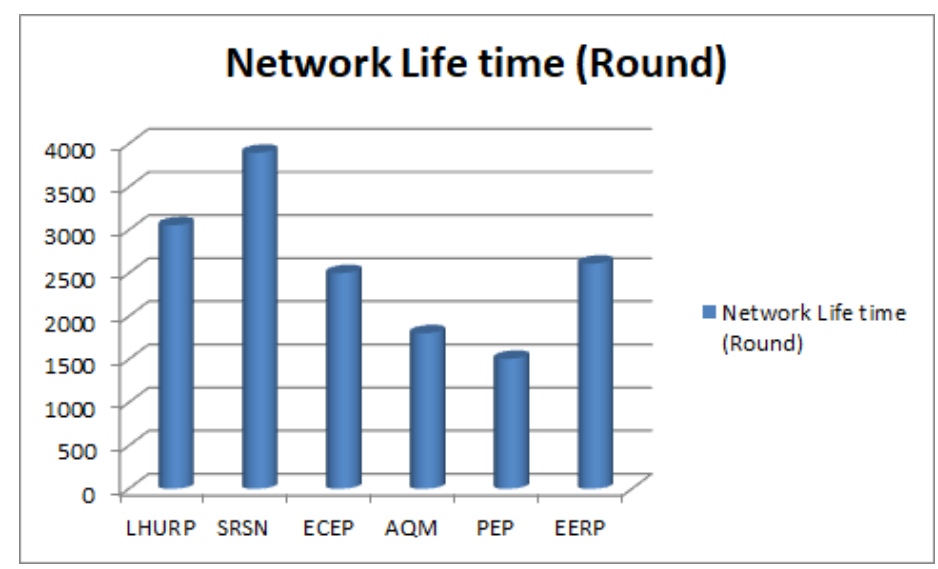

Figure 5 Network Life Time Vs Protocol

Figure 5 shows the performance measurements of proposed methods between network lifetime vs. protocol. The lifetime of the network can be defined as the time between the beginning and the end of the network node. The energy efficiency is very high in EERP protocol in WSN, which is used to reduce the energy consumption rate in the data routing.

ISSN: 2582-1369 (online)

Submitted: 23.04 .2021

Revised: 5.05.2021

Accepted: 20.05.2021

Published: 3.06.2021 
Table 1 Overall Performance Metric Measures of Various Proposed Protocols

\begin{tabular}{|c|c|c|c|c|c|c|c|c|c|}
\hline S.No & Protocol Model & $\begin{array}{c}\text { Data } \\
\text { Aggregation }\end{array}$ & Scalability & $\begin{array}{c}\text { Power } \\
\text { Usage }\end{array}$ & Reliability & $\begin{array}{c}\text { Congestion } \\
\text { Control }\end{array}$ & $\begin{array}{c}\text { Energy } \\
\text { Efficiency }\end{array}$ & $\begin{array}{c}\text { Delivery } \\
\text { Delay }\end{array}$ & $\begin{array}{c}\text { Network } \\
\text { Lifetime }\end{array}$ \\
\hline 1 & LHURP & NA & Medium & NA & Medium & High & NA & Low & High \\
\hline 2 & ECRP & NA & Medium & NA & Medium & High & NA & Low & High \\
\hline 3 & AQM & Medium & Low & Low & NA & High & Medium & Very Large & Low \\
\hline 4 & SRSN & NA & Low & NA & NA & Medium & Low & NA & Very Large \\
\hline 5 & EERP & NA & Low & Low & NA & NA & High & Medium & High \\
\hline 6 & ECEP & Medium & NA & NA & Low & NA & NA & Large & Medium \\
\hline 7 & PEP & NA & Low & Low & Low & NA & Low & Very Large & Low \\
\hline
\end{tabular}

The main aim of routing protocol is to maximize the network lifetime while maintaining the network quality. The most energy-efficient task in WSN is data routing. Thus, intelligent techniques must be used to reduce the usage of energy in the routing protocol.

\section{CONCLUSION}

In recent years, researchers have been motivated by the emergence of WSNs for wide range of purposes by performing routing tasks among the most active and challenging research domains in WSN projects. The proposed protocols have investigated the recent taxonomy and has suggested 9 categories of protocols: type of application, mode of delivery, communication initiator, network architecture, path-development (route discovery), and network topology (structure), operation of the protocol, next-hop selection, latency warning, and effective path detection. In addition, under the NS3 simulator, performance assessment has been carried out on the most representative protocols. As a future work, the security measures of WSN can be developed through the simulation analysis performed by intelligent function procedure [39]. In this research paper, the application-related interplay between these ingredients and energy were ignored when the research work aims to draw energy-consuming components and their relations in a generic model. One extension to the architecture would therefore be to explore energy 
consumption patterns and shapes for a generic application (e.g. health monitoring applications) and to develop a complete energy consumption map for a particular application. One additional goal is to produce the whole network of wireless sensors with a single overall energy consumption formula; a first examination took the weighted, linear energy consumption with a combination of the components. Components can interact as a function of the WSN and application in terms of their weights.

In the future, a more accurate energy costs function will be developed with due emphasis on parameters, components, and playoff factors for components. The energy cost function should therefore be considered [40]. We think that a nonlinear cost function would allow the model to better adapt to a specific WSN application rather than a linear combination. A second important purpose that has been planned to pursue in the next phase is to fully model the components of each of the architecture's energy components.

\section{REFERENCES}

[1] Tang, L.; Lu, Z.; Fan, B. Energy Efficient and Reliable Routing Algorithm for Wireless Sensors Networks. Appl. Sci. 2020, 10, 1885.

[2] Zhao, Z.; Hou, M.; Zhang, N.; Gao, M. Multipath Routing Algorithm Based on Ant Colony Optimization and Energy Awareness. Wirel. Pers. Commun. Int. J. 2017, 94, 2937-2948.

[3] Sun, Y.; Dong,W.; Chen, Y. An Improved Routing Algorithm Based on Ant Colony Optimization inWireless Sensor Networks. IEEE Commun. Lett. 2017, 21, 1317-1320.

[4] Yu, S.; Zhang, B.; Li, C.; Mouftah, H.T. Routing protocols for wireless sensor networks with mobile sinks: A survey. IEEE Commun. Mag. 2014, 52, 150-157.

[5] Radi, M.; Dezfouli, B.; Bakar, K.A.; Lee, M. Multipath routing in wireless sensor networks: Survey and research challenges. Sensors 2012, 12, 650-685.

[6] Radi, M.; Dezfouli, B.; Abd Razak, S.; Bakar, K.A. LIEMRO: A Low-Interference energyefficient multipath routing protocol for improving QoS in event-based wireless sensor networks. In Proceedings of the 2010 Fourth International Conference on Sensor Technologies and Applications, Venice, Italy, 18-25 July 2010; pp. 551-557. 
[7] Jayashree, A.; Biradar, G.S.; Mytri, V.D. Review of Multipath Routing Protocols in Wireless Multimedia Sensor Network-A Survey. Int. J. Sci. Eng. Res. 2012, 3, 1-9.

[8] Deng, S.; Li, J.; Shen, L. Mobility-based clustering protocol for wireless sensor networks with mobile nodes. IET Wirel. Sens. Syst. 2011, 1, 39-47.

[9] Awwad, S.A.; Ng, C.K.; Noordin, N.K.; Rasid, M.F. Cluster based routing protocol for mobile nodes in wireless sensor network. Wirel. Pers. Commun. 2011, 61, 251-281.

[10] Rai, D.; Hiwale, A.S. Enhanced MBC (mobility-based clustering) protocol for wireless AD-hoc networks. Int. J. Eng. Sci. Innov.Technol. 2014, 3, 171-175.

[11] Rathee, A.; Kashyap, I.; Choudhary, K. Developed Distributed Energy-Efficient Clustering (DDEEC) Algorithm based on Fuzzy Logic Approach for Optimizing Energy Management in Heterogeneous WSNs. Int. J. Comput. Appl. 2015, 115, 8887.

[12] Kashaf, A.; Javaid, N.; Khan, Z.A.; Khan, I.A. TSEP: Threshold-sensitive stable election protocol for WSNs. In Proceedings of the 2012 10th International Conference on Frontiers of Information Technology, Islamabad, India, 17-19 December 2012; pp. 164-168.

[13] Kumar, S.; Verma, S.K.; Kumar, A. Enhanced Threshold Sensitive Stable Election Protocol for HeterogeneousWireless Sensor Network. Wirel. Pers. Commun. 2015, 85, 2643-2656.

[14] Javaid, N.; Qureshi, T.N.; Khan, A.H.; Iqbal, A.; Akhtar, E.; Ishfaq, M. EDDEEC: Enhanced developed distributed energy-efficient clustering for heterogeneous wireless sensor networks. Procedia Comput. Sci. 2013, 19, 914-919.

[15] Sharma, E.R.; Sharma, E.S. A Survey on Various Routing Protocols in Wireless Sensor Networks. Int. J. Innov. Eng. Technol. 2016, 7, 486-493.

[16] Qureshi, T.N.; Javaid, N.; Khan, A.H.; Iqbal, A.; Akhtar, E.; Ishfaq, M. BEENISH: Balanced energy efficient network integrated super heterogeneous protocol for wireless sensor networks. Procedia Comput Sci. 2013, 19, 920-925.

[17] WANG, Q., HEMPSTEAD, M. \& YANG, W. A Realistic Power Consumption Model for Wireless Sensor Network Devices. Third Annual IEEE Communications Society Conference on Sensor, Mesh and Ad Hoc Communications and Networks (IEEE SECON 2006), 2006a VA, USA. IEEE, 286-295. 
[18] Kulik, J.; Heinzelman, W.; Balakrishnan, H. Negotiation-based protocols for disseminating information in wireless sensor networks. Wirel. Netw. 2002, 8, 169-185.

[19] GOLDSMITH, A. J. \& WICKER, S. B. 2002. Design Challenges for Energy-Constrained Ad Hoc Wireless Networks. IEEE Wireless Communications Magazine,, 8-27.

[20] MUSZNICKI, B., TOMCZAK, M. \& ZWIERZYKOWSKI, P. Dijkstra-based Localized Multicast Topology management in Wireless Sensor Networks. 8th IEEE, IET International Symposium on Communication Systems, Networks and Digital Signal Processing, 2012.

[21] BHATTACHARYA, A. \& KUMAR, A. 2014. A shortest path tree based algorithm for relay placement in a wireless sensor network and its performance analysis. Comput. Netw., 71, 48-62.

[22] QIONG, H., BO, D. \& SUBIR, B. 2013. Pulse Switching. Wireless Sensor Networks. CRC Press.

[23] Verma, S.; Sood, N.; Sharma, A.K. A novelistic approach for energy efficient routing using single and multiple data sinks in heterogeneous wireless sensor network. Peer-Peer Netw. Appl. 2019, 12, 1110-1136.

[24] Harous, S.; Aliouat, Z. Energy Efficient Multi-Hops Clustering Protocol forWireless Sensor Networks. Int. J. Comput. Commun. 2015, 9, 88-95.

[25] KC, K.P.; Terence, S. A Survey on Event Detection and Transmission Protocols in an Event Driven Wireless Sensor Network. Int. J. Comput. Appl. 2012, 58, 12-18.

[26] Mahajan, A.S.; Dhamdhere, V. Energy Efficient Fast Forwarding in Event Driven Wireless Sensor Network (EWSN) using Route Discovery. Energy 2013, 2, 1-7.

[27] Sangeetha, K. Fast, Reliable and Efficient Event-Detecting (Freed) Protocol for EventDrivenWireless Sensor Networks. Int. J. Comput. Appl. 2013, 10, 1-6.

[28] Abazeed, M.; Faisal, N.; Zubair, S.; Ali, A. Event driven routing protocols for wireless sensor network-A Survey. Int. J. Comput. Sci. Appl. 2013.

[29] Nigam, G.K.; Dabas, C. "A Survey on Protocols and Routing Algorithms for Wireless Sensor Networks" In Proceedings of the World Congress on Engineering and Computer Science, San Francisco, CA, USA, 21-23 October 2015. 
[30] Roseline, R.A.; Sumathi, P. Energy efficient routing protocols for wireless sensor networks: A survey. Int. J. Comput. Appl. 2017, 165, 41-46.

[31] Kochhar, A.; Kaur, P.; Singh, P.; Sharma, S. Protocols forWireless Sensor Networks: A Survey. J. Telecommun. Inf. Technol. 2018, 77-87.

[32] Nakas, C.; Kandris, D.; Visvardis, G. Energy Efficient Routing in Wireless Sensor Networks: A Comprehensive Survey. Algorithms 2020, 13, 72.

[33] Kardi, A.; Zagrouba, R.; Alqahtani, M. "A taxonomy of routing protocols in Wireless Sensor Networks" In Proceedings of the $20^{\text {th }}$ International Conference onWireless Information Technology and Systems, Lisbon, Portugal, 4 September 2018.

[34] Sabor, N.; Sasaki, S.; Abo-Zahhad, M.; Ahmed, S.M. A Comprehensive Survey on Hierarchical-Based Routing Protocols for MobileWireless Sensor Networks: Review, Taxonomy, and Future Directions. Wirel. Commun. Mob. Comput. 2017, 2017, 1-23.

[35] Jaber, G.; Kacimi, R. “A collaborative caching strategy for content-centric enabled wireless sensor networks" Comput. Commun. 2020, 159, 60-70.

[36] Chandirika, B.; Sakthivel, N.K. Performance Analysis of Clustering-Based Routing Protocols forWireless Sensor Networks. In Advances in Big Data and Cloud Computing; Springer: Singapore, 2018; pp. 269-276.

[37] Pandey, M.A.; Gupta, P.N.; Vardhan, H. Performance Evaluation of Various Routing Protocols and quality of service for Wireless Sensor Network. J. Telecommun. Stud. 2018, 4, 24-35.

[38] Goyal, D.; Tripathy, M.R. Routing Protocols in Wireless Sensor Networks: A Survey. In Proceedings of the 2012 Second International Conference on Advanced Computing \& Communication Technologies, Rohtak, Haryana, India, 7-8 January 2012.

[39] Rathi, N.; Saraswat, J.; Bhattacharya, P.P. A review on routing protocols for application in wireless sensor networks. arXiv 2012, arXiv:1210.2940.

[40] Krishnaveni, P.; Sutha, J. Analysis of routing protocols for wireless sensor networks. Int. J. Emerg. Technol. Adv. Eng. 2012, 2, 401-407. 